\title{
MODULAÇÃO AUTONÔMICA DA FREQUÊNCIA CARDÍACA E CAPACIDADE FUNCIONAL AERÓBICA EM HEMIPARÉTICOS
}

\author{
AUTONOMIC HEARTRATEMODULATIONAND FUNCTIONALAEROBIC CAPACITY IN HEMIPARETICPATIENTS \\ MODULACIÓN AUTONÓMICA DE LAFRECUENCIA CARDÍACA Y CAPACIDAD FUNCIONAL AERÓBICA EN \\ HEMIPARÉTICOS
}

Mônica Furquim de Campos' (Fisioterapeuta)

Suenimeire Vieira² (Fisioterapeuta)

Alexandre Ricardo Pepe Ambrozin ${ }^{3}$ (Fisioterapeuta)

Aparecida Maria Catai ${ }^{4}$

(Fisioterapeuta)

Robison José Quitério ${ }^{1,3,4}$

(Fisioterapeuta)

1. Departamento de Educação Física, Instituto de Biociências, Universidade Estadual Paulista "Júlio de Mesquita Filho". Campus de Rio Claro, SP, Brasil.

2. Departamento de Biomecânica, Medicina e Reabilitação do Aparelho Locomotor, Universidade de São Paulo. Campus de Ribeirão

Preto, SP, Brasil.

3. Departamento de Fisioterapia e Terapia Ocupacional, Universidade Estadual Paulista "Júlio de Mesquita Filho". Campus de Marília, SP, Brasil.

4. Departamento de Fisioterapia, Centro de Ciências Biológicas e da Saúde, Universidade Federal de São Carlos. São Carlos, SP, Brasil.

\section{Correspondência:}

Robison José Quitério. Av. Higyno Muzzi Filho, 737, Cidade Universitária, Marília, SP, Brasil. 17525-900.

robisonq@gmail.com

\section{RESUMO}

Introdução:Tem sido observado que indivíduos acometidos por acidente vascular encefálico (AVE) apresentam, além dos danos físicos, diminuição da capacidade aeróbica e alteração da modulação autonômica da frequência cardíaca, sendo estes importantes fatores para o aparecimento de doenças cardíacas e arritmias. Objetivo: Investigar os efeitos crônicos do AVE sobre a capacidade aeróbica $\left(\mathrm{VO}_{2 \text { pico }}\right)$ e os índices de variabilidade da frequência cardíaca (VFC), e se existe correlação entre essas duas variáveis. Métodos: Foram avaliados 11 indivíduos do sexo masculino, com idade entre 55 e 65 anos, acometidos por lesão cerebrovascular há pelo menos seis meses, e todos com hemiparesia. Foram realizadas as seguintes avaliações: teste de capacidade aeróbica máxima; registro dos intervalos R-R e cálculo dos índices da VFC (RMSSD, pNN50, AF, BF e a razão BF/AF). Para comparação entre $V_{2} O_{2 \text { ico }}$ previsto e obtido utilizou-se o teste t de Student não pareado. Para testar a hipótese de correlação entre os índices de VFC e o VO 2 pico utilizou-se o teste de correlação de Pearson. Resultados: $\mathrm{VO}_{2 \text { pico }}\left(\mathrm{mL} \mathrm{kg}^{-1} \cdot \mathrm{min}^{-1}\right)$ previsto e obtido, respectivamente $=32,15 \pm 1,87$ e 16,12 $\pm 5,51$; índices da VFC: RMSSD (ms) $=28,69 \pm 26,78$; pNN50 (\%) $=8,76 \pm 12,62 ; \mathrm{AF}$ (u.n.) $=51,96 \pm 22,4 ; \mathrm{BF}$ (u.n.) $=48,04 \pm 22,49 . \mathrm{OVO}_{2 \text { pico }}$ correlacionou-se negativamente com os índices RMSSD, pNN50 e AF e positivamente com o índice $B F(p<0,05)$. Conclusão: Na fase crônica do AVE, variabilidade da frequência cardíaca encontra-se normal e os indivíduos com menor capacidade aeróbica apresentam maior modulação parassimpática e menor simpática, provavelmente em consequência da maior exigência física nas atividades de vida diária.

Descritores: acidente vascular cerebral; frequência cardíaca; consumo de oxigênio; sistema nervoso autônomo.

\section{ABSTRACT}

Introduction: It has been observed that individuals affected by stroke have, in addition to the physical damage, decreased aerobic capacity and impaired heart rate variability, which are important factors for the onset of cardiac diseases and arrhythmias. Objective: To investigate the chronic effects of stroke on aerobic capacity $\left(\mathrm{VO}_{2 \text { peak }}\right)$ and the indices of heart rate variability (HRV), and if there is a correlation between these two variables. Methods: Eleven male individuals aged 55 to 65 affected by cerebrovascular injury for at least six months all with hemiparesis were assessed. The following evaluations were performed: maximum aerobic capacity test, record of $R$-R intervals and calculation of the indices of HRV (RMSSD, pNN50, HF, LF and LF/HF ratio). For the comparison between expected and obtained V $\mathrm{O}_{2 \text { peak }}$ the unpaired Student's $t$-test was used. To test the hypothesis of correlation between HRV indices and V $\mathrm{O}_{\text {2peak }}$ the Pearson correlation test was used. Results: $\mathrm{VO}_{2 \text { peak }}\left(\mathrm{mL} . \mathrm{kg}^{-1} . \mathrm{min}^{-1}\right)$ expected and obtained, respectively $=32.15 \pm 1.87$ and $16.12 \pm 5.51$; HRV indices: RMSSD (ms) $=28.69 \pm 26.78 ; p N N 50(\%)=8.76 \pm 12.62 ; \mathrm{HF}$ (n.u. $)=51.96 \pm 22.4 ; \mathrm{LF}($ n.u. $)=48.04 \pm 22.49$. The $V^{2}{ }_{2 \text { peak }}$ correlated negatively with the RMSSD, pNN50 and HF indices; and positively with LF $(p<0.05)$. Conclusion: In the chronic phase of stroke, the heart rate variability is normal and individuals with lower aerobic capacity have higher parasympathetic modulation and lower sympathetic modulations, probably due to the higher physical demand on physical activities of daily living.

Keywords: stroke; heart rate; oxygen consumption; autonomic nervous system.

\section{RESUMEN}

Introducción: Se ha observado que los individuos afectados por accidente cerebrovascular (ACV) presentan, además de los daños físicos, disminución de la capacidad aeróbica y alteración de la modulación autonómica cardiaca, que son factores importantes para la aparición de la enfermedad cardiaca y arritmias. Objetivo: Investigar los efectos crónicos del ACV sobre la capacidad aeróbica $\left(V_{2} \mathrm{p}_{\text {picd }}\right.$ y los índices de variabilidad del ritmo cardíaco (VRC), y si existe una correlación entre estas dos variables. Métodos: Se evaluaron 11 sujetos del sexo masculino de 55 a 65 años de edad afectados por una lesión cerebrovascular durante al menos seis meses, todos con hemiparesia. Se realizaron las siguientes evaluaciones: prueba de capacidad aeróbica máxima; registro de intervalos $R$ - $R$ y el cálculo de los índices de VRC (RMSSD, pNN50, AF, BF y la razón BF/AF). Para la comparación entre VO 2 2pico esperado y obtenido se utilizó la prueba de la t no pareada. Para probar la hipótesis de correlación entre los índices de VRC y $\mathrm{VO}_{2 \text { pico }}$ se utilizó el coeficiente de correlación de Pearson. Resultados: $\mathrm{VO}_{2 \text { pico }}\left(\mathrm{mL} \mathrm{kg}^{-1} . \mathrm{min}^{-1}\right)$ esperado y obtenido, respectivamente $=32,15 \pm 1,87$ y 16,12 $\pm 5,51$; indices de VRC: $\operatorname{RMSSD}(\mathrm{ms})=28,69 \pm 26,78 ;$ pNN50 (\%) $=8,76 \pm 12,62$; 
$A F$ (u.n.) $=51,96 \pm 22,4 ; B F$ (u.n.) $=48.04 \pm 22.49$. El $V_{2 \text { 2pico }}$ se correlacionó negativamente con los índices RMSSD, pNN50 y AF y positivamente con el índice BF $(p<0,05)$. Conclusión: En la fase crónica del ACV, la variabilidad del ritmo cardíaco es normal y las personas con menor capacidad aeróbica tienen modulación parasimpática mayor y simpática menor, probablemente como resultado de una mayor demanda física en las actividades de la vida diaria.

Descriptores: accidente cerebrovascular; frecuencia cardíaca; consumo de oxígeno; sistema nervioso autónomo.

Artigo recebido em 24/06/2015 aprovado em 18/08/2016.

\section{INTRODUÇÃO}

A prevalência do acidente vascular encefálico (AVE) tem aumentado consideravelmente nos últimos anos em decorrência do enveIhecimento populacional e a maior taxa de sobrevida após o evento vascular ${ }^{1}$. Neste sentido, têm sido necessário maiores investigações sobre as suas repercussões orgânicas.

As alterações motoras decorrentes, tais como diminuição da força, da massa muscular, da eficiência e velocidade dos movimentos, somadas aos distúrbios de contração, de tônus muscular, de reflexos e de controle postural prejudicam a função musculoesquelética ${ }^{2}$, reduzindo as atividades de vida diária ${ }^{3}$ e aumentando o gasto energético em um e meio a duas vezes para a mesma atividade quando comparado a indivíduos saudáveis ${ }^{4}$.

Adicionalmente, a extensão da lesão, o hemisfério cerebral acometido, as comorbidades cardiovasculares e os medicamentos em uso ${ }^{5}$ contribuem para a redução de cerca de 50\% a 75\% da capacidade aeróbica ${ }^{2,6}$, além de causar danos ao miocárdio, aumentar a incidência de arritmias e prejudicar o controle autonômico do coração ${ }^{7,8}$.

Assim, tem sido observado que, além dos danos físicos e da diminuição da capacidade aeróbica, ocorre alteração na modulação autonômica da frequência cardíaca, tanto na fase aguda quanto na fase crônica do AVE'.

Considerando que alguns estudos estão de acordo que, indivíduos com maior capacidade aeróbica podem apresentar melhor controle neurocardíaco ${ }^{10}$, possivelmente devido ao aumento da atividade vagal ${ }^{11}$ e/ou diminuição da atividade simpática ${ }^{12}$, o presente estudo teve como objetivo investigar os efeitos crônicos do AVE sobre a capacidade aeróbica $\left(\mathrm{VO}_{2 \text { pico }}\right)$ e os índices de variabilidade da frequência cardíaca (VFC) e se existe correlação entre essas duas variáveis; se existe associação entre hemisfério cerebral acometido e medicamentos em uso com índices de VFC e oVO ${ }_{2 \text { pico. }}$ Neste sentido a hipótese deste estudo é que indivíduos com maior capacidade aeróbica apresentem melhores índices de variabilidade da frequência cardíaca, independente do hemisfério cerebral acometido e uso de medicamentos.

\section{MATERIAIS E MÉTODOS}

Foram triados 73 voluntários no serviço de neurologia da Clínica de Fisioterapia da Universidade Estadual Paulista (UNESP), Marília/SP, Brasil, e das Unidades Básicas de Saúde do município de Marília/SP, Brasil, porém apenas 11 preencheram todos os critérios de inclusão e compuseram esse estudo: Homens, não praticantes de atividade física regular, não fumantes, com idade entre 55 e 65 anos, acometidos por lesão cerebrovascular há no mínimo seis meses, com hemiparesia e exame ecocardiográfico sem alterações. Foram excluídos aqueles que apresentaram doenças respiratórias; disfunção neurológica e/ou cardiovascular prévia ao AVE; plegia em qualquer segmento corporal; distúrbios psiquiátricos e/ou cognitivos que impediriam a coleta dos dados, sujeitos tabagistas e com contra indicação a realização do Teste de Esforço Físico Aeróbico. A Figura 1 representa de forma detalhada os meios de contato com os voluntários, divulgação do estudo e respectiva perda amostral.
O estudo foi transversal, aprovado pelo Comitê de Ética em Pesquisa com seres humanos (parecer 0093/2011). Depois de realizada a triagem, os voluntários foram informados sobre os objetivos do trabalho e os procedimentos experimentais e assinaram o Termo de Consentimento Livre e Esclarecido conforme determina a resolução 466/12e suas normas complementares do Conselho Nacional de Saúde.

As avaliações foram realizadas em ambiente silencioso e climatizado $\left(23 \pm 2^{\circ} \mathrm{C}\right)$, no período da manhã e constaram de anamnese, coleta de dados sobre a história pregressa do voluntário, medidas da pressão arterial (PA) e frequência cardíaca (FC), ausculta pulmonar e cardíaca, medidas da massa corporal e estatura, cálculo do índice de massa corpórea (IMC), teste ergométrico (TE), registro da FC e dos intervalos RR (iRR) e ecocardiograma.

Em dias diferentes, os voluntários retornaram para a realização do TE e registro da $\mathrm{FC}$ e dos intervalos $\mathrm{RR}$ (iRR). Os voluntários não ingeriram café, chá, refrigerante e bebidas alcoólicas, e mantiveram as atividades de vida diária nas 48 horas anteriores aos testes.

OTE foi realizado em um cicloergômetro (Inbramed, RS, Brasil), aplicando-se o protocolo de Balke que consta do incremento de 25 watts de carga a cada estágio de três minutos. No final de cada e/stágio foram coletados: sinal do eletrocardiógrafo por meio de um monitor cardíaco de um canal (Ecafix, Brasil), FC, PA e índice de percepção de esforço para cansaço cardiorrespiratório e de membros inferiores por meio da escala de Borg modificada. Foram adotados os critérios da Sociedade Brasileira de Cardiologia para a interrupção do TE sintoma limitado.

O consumo de oxigênio máximo previsto e o obtido no pico do esforço físico foram calculados aplicando-se as seguintes fórmulas: $\mathrm{VO}_{2}$ previsto $=57,8-(0,445 \times \text { idade })^{13} ; \mathrm{VO}_{2 \text { pico }}=($ carga máxima atingida em Watts $\times 12)=300 /$ massa corporal ${ }^{14}$.

A FC e os iRR instantâneos foram gravados por um sistema digital de telemetria validado previamente que consiste de um transmissor posicionado na altura do processo xifoide e um monitor / receptor (Polar RS800CX, Polar ElectroOy, Kempele, Finland) ${ }^{15}$.

Para o registro da FC e dos intervalos RR (iRR), o sujeito foi posicionado em decúbito dorsal e permaneceu em repouso até que as variáveis fisiológicas se estabilizassem (PA e FC). A partir desse momento e nessa postura foi realizado o registro durante 10 minutos.

Os dados foram transmitidos para um computador utilizando-se o software Polar Precision Performance (versão 3.02.007) e convertidos em arquivos de texto que foram analisados no Software Kubios HRV

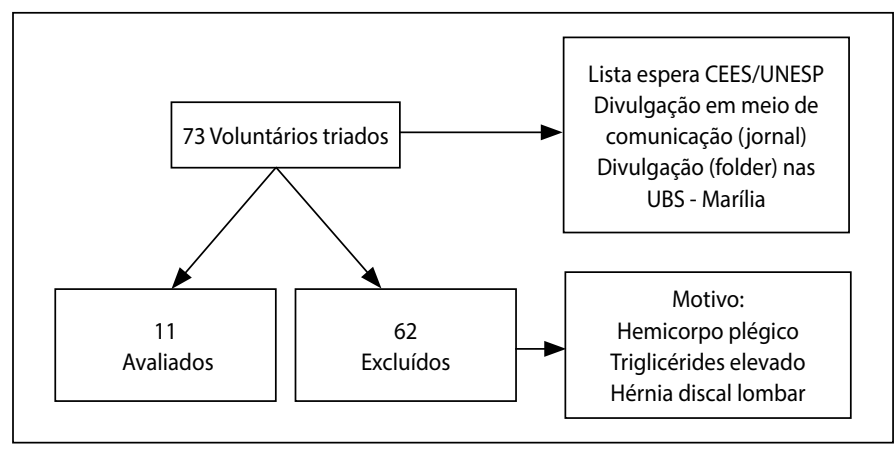

Figura 1. Fluxograma de perda amostral. 
(versão 2.0, University of Kuopio, Finland). Foi aplicado filtro digital de baixa intensidade para eliminar os intervalos RR anormais, e selecionado o trecho mais estável para análise, sempre considerando 256 pontos. Foram utilizados índices estatísticos no domínio do tempo, RMSSD (raiz quadrada da média do quadrado das diferenças entre intervalos RR normais adjacentes, em um intervalo de tempo expresso em milissegundos) e pNN50 (porcentagem dos intervalos RR adjacentes com diferença de duração maior que 50 milissegundos); e no domínio da frequência, que decompõe o sinal nas seguintes bandas: alta frequência ( $A F-0,15$ a $0,4 \mathrm{~Hz}$ ) que se associa a atividade parassimpática; baixa frequência ( $\mathrm{BF}-0,04$ a $0,15 \mathrm{~Hz}$ ) que representa atividade simpática e parassimpática, porém com o predomínio da simpática; e a razão BF/AF que representa o balanço simpato vagal. Os índices de VFC são apresentados em milissegundos ao quadrado $\left(m s^{2}\right)$ e em unidades normalizadas (u.n. $)^{16}$.

\section{Análise estatística}

O cálculo amostral foi realizado por meio do software Ene ${ }^{\oplus}$, versão 3.0 (Barcelona, Espanha). Considerou-se a correlação entre as variáveis AF e $\mathrm{VO}_{2}$ realizada em estudo piloto. Assim, utilizou-se $r=0,62$, poder estatístico de $80 \%$ e alfa de 0,05 . Com estes parâmetros, determinou-se um tamanho de amostra de 15 voluntários. Aplicou-se o teste de Kolmogorov-Smirnov para verificar a distribuição paramétrica da amostra.

Os dados demográficos, antropométricos e fisiológicos são apresentados de forma descritiva em média e desvio padrão. Para comparação entre os valores de $\mathrm{VO}_{2 \text { pico }}$ previsto e obtido foi utilizado o teste $t$-Student não pareado. Para testar a hipótese de correlação entre os índices de VFC com - $\mathrm{VO}_{2 \text { pico }}$ foi aplicado o teste de Pearson Correlation. E para análise de associação entre as variáveis $\mathrm{VO}_{2 \text { pico }}$ e índices de VFC com o hemisfério cerebral acometido e os medicamentos em uso foi aplicado o Fisher's Exact Test.

O nível de significância adotado foi de $5 \%(p \leq 0,05)$. Foi utilizado o software de análise GraphPad InStat ${ }^{\oplus}$ (Califórnia, EUA).

\section{RESULTADOS}

A amostra consistiu de 11 homens, sendo seis com hemiparesia à direita e cinco à esquerda, sem alterações clínicas e ecocardiográficas. As características da amostra, comorbidades e os medicamentos em vigência são apresentados na Tabela1.

Todos os testes ergométricos foram interrompidos por fadiga do membro inferior não parético.

O consumo de oxigênio máximo previsto e o obtido no pico do exercício, os índices da VFC no domínio do tempo (iRR, RMSSD, pNN50) e no domínio da frequência ( $A F, B F, B F / A F)$ em repouso estão apresentados na Tabela 2. $\mathrm{O} \mathrm{VO}_{2}$ previsto e obtido apresentaram resultados significativos $(p<0,0001)$.

Para os valores de $\mathrm{VO}_{2 \mathrm{pico}}$, houve correlação significativa $(p<0,05)$ negativa com os índices RMSSD, pNN50 e AF, e positiva com o índice BF (Figuras 2, 3, 4 e 5).

O teste de associação entre os índices da VFC e VO $\mathrm{Z}_{2 \text { pico }} \mathrm{com}$ os medicamentos em uso e com o hemisfério cerebral acometido não apresentaram significância estatística ( $p \geq 0,05)$.

\section{DISCUSSÃO}

Os principais achados do presente estudo foram: 1) $\circ \mathrm{VO}_{2 \text { pico }}$ apresentou déficit de 48,68土16,91\%; 2) os valores dos índices de VFC apresentaram-se dentro da faixa de normalidade referida pela literatura; 3) o $\mathrm{VO}_{2 \text { pico }}$ correlacionou-se negativamente com os índices que representam a modulação parassimpática e positivamente com o índice associado a modulação simpática; 4) Não foi verificada associação entre modulação autonômica da frequência cardíaca ou do $\mathrm{VO}_{2 \text { pico }} \mathrm{com}$ o uso de medicamentos ou com o hemisfério cerebral acometido.
Como verificado por outros estudos ${ }^{17,18}$, os dados indicam que o comprometimento motor foi o fator limitante do teste de esforço físico aeróbico, sendo responsável pelas respostas das variáveis cardiovasculares ficarem bem abaixo dos valores esperados, já que não foram identificadas alterações na avaliação clínica do aparelho cardiorrespiratório

Tabela 1. Características da amostra, comorbidades e medicamentos utilizados pelos indivíduos ( $n=11)$.

\begin{tabular}{|c|c|}
\hline Dados antropométricos e fisiológicos & Média $\pm \mathrm{DP}$ \\
\hline Idade (anos) & $57,63 \pm 4,20$ \\
\hline Estatura $(\mathrm{m})$ & $1,72 \pm 0,08$ \\
\hline Massa Corporal (Kg) & $81,86 \pm 15,40$ \\
\hline IMC $\left(\mathrm{Kg} / \mathrm{m}^{2}\right)$ & $27,47 \pm 4,78$ \\
\hline PAS $(\mathrm{mmHg})$ & $129,55 \pm 12,34$ \\
\hline PAD (mmHg) & $82,73 \pm 11,91$ \\
\hline FC de repouso (bpm) & $6,37 \pm 7,30$ \\
\hline Tempo de AVE (anos) & $3,78 \pm 3,08$ \\
\hline Fatores de risco & $\%$ \\
\hline Dislipidemia & 36,36 \\
\hline Hiperglicemia & 27,27 \\
\hline Hipertensão & 81,82 \\
\hline Medicamentos & $\%$ \\
\hline Hipolipimiantes & 36,36 \\
\hline Hipoglicemiante & 27,27 \\
\hline Inibidor da enzima conversora de angiotensina & 36,36 \\
\hline Bloqueador dos canais de cálcio & 18,18 \\
\hline Beta bloqueador & 9,09 \\
\hline Diuréticos & 54,55 \\
\hline Antagonistas dos receptores de angiotensina & 27,27 \\
\hline Anticoagulantes & 72,73 \\
\hline Agonistas adrenérgicos & 9,09 \\
\hline
\end{tabular}

Tabela 2. Consumo de oxigênio máximo previsto e obtido no pico do esforço, índices da variabilidade da frequência cardíaca no domínio do tempo (iRR, RMSSD, pNN50) e no domínio da frequência (BF, AF, BF/AF).

\begin{tabular}{|c|c|}
\hline & Média $\pm \mathrm{DP}$ \\
\hline $\mathrm{VO}_{2}$ previsto $\left(\mathrm{mL} \mathrm{Kg}^{-1} \cdot \mathrm{min}^{-1}\right)$ & $32,15 \pm 1,87$ \\
\hline $\mathrm{VO}_{2}$ pico $\left(\mathrm{mL} \cdot \mathrm{Kg}^{-1} \cdot \mathrm{min}^{-1}\right)$ & $16,12 \pm 5,51$ \\
\hline Intervalo RR (iRR) & $0,875 \pm 0,09$ \\
\hline RMSSD (ms) & $28,69 \pm 26,78$ \\
\hline pNN50 (\%) & $8,76 \pm 12,62$ \\
\hline Baixa frequência (BF - u.n.) & $48,04 \pm 22,49$ \\
\hline Baixa frequência $\left(B F-m s^{2}\right)$ & $231 \pm 203,87$ \\
\hline Alta frequência (AF - u.n.) & $51,96 \pm 22,49$ \\
\hline Alta frequência $\left(\mathrm{AF}-\mathrm{ms}^{2}\right)$ & $414,45 \pm 651,5$ \\
\hline $\mathrm{BF} / \mathrm{AF}$ & $1,67 \pm 1,95$ \\
\hline
\end{tabular}

$\mathrm{VO}_{2}$ : consumo de oxigênio; RMSSD: raiz quadrada da média do quadrado das diferenças entre iRR normais adjacentes; pNN50: porcentagem dos iRR adjacentes com diferença de duração maior que 50 milissegundos.

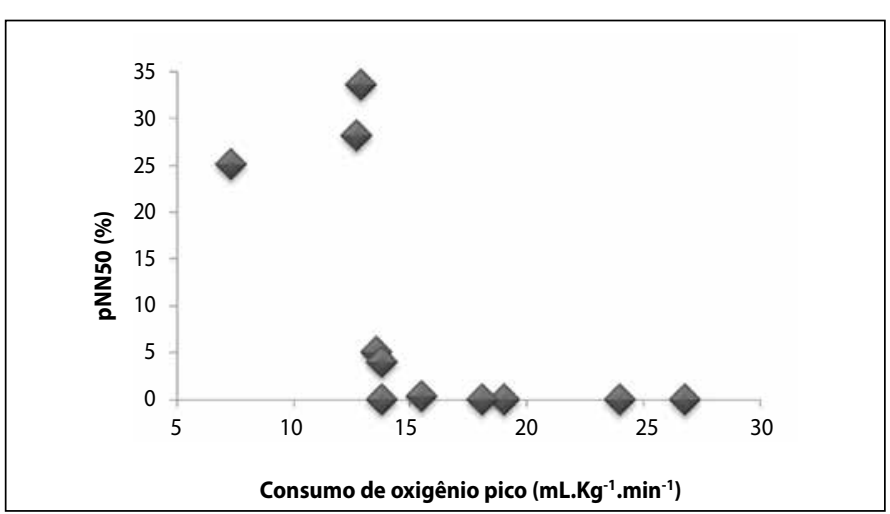

Figura 2. Dispersão do índice de variabilidade da frequência cardíaca no domínio do tempo (pNN50) e do consumo pico de oxigênio $(n=11) r=-0,61$ e $p=0,04$. 


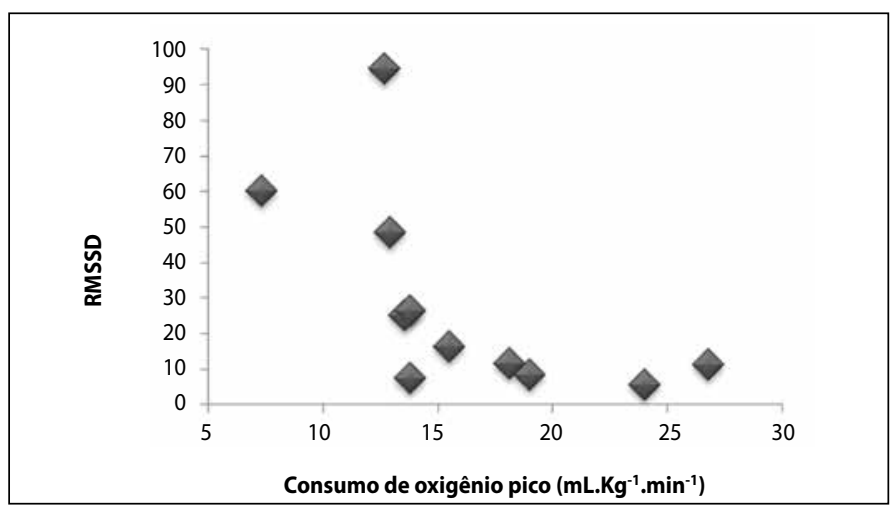

Figura 3. Dispersão do índice de variabilidade da frequência cardíaca no domínio do tempo (RMSSD) e do consumo pico de oxigênio $(n=11) r=-0,61$ e $p=0,04$.

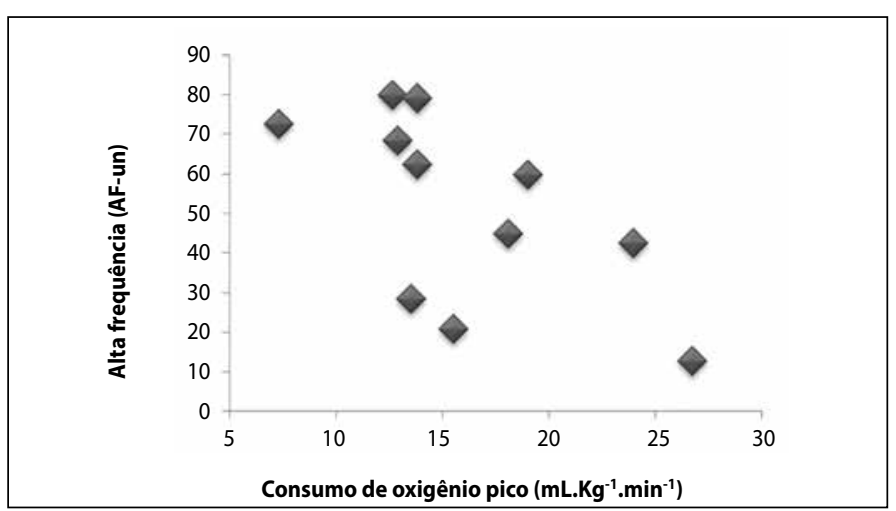

Figura 4. Dispersão do índice espectral de alta frequência (AF - 0,15 a 0,4Hz) e do consumo pico de oxigênio $(n=11) r=-0,63$ e $p=0,03$.

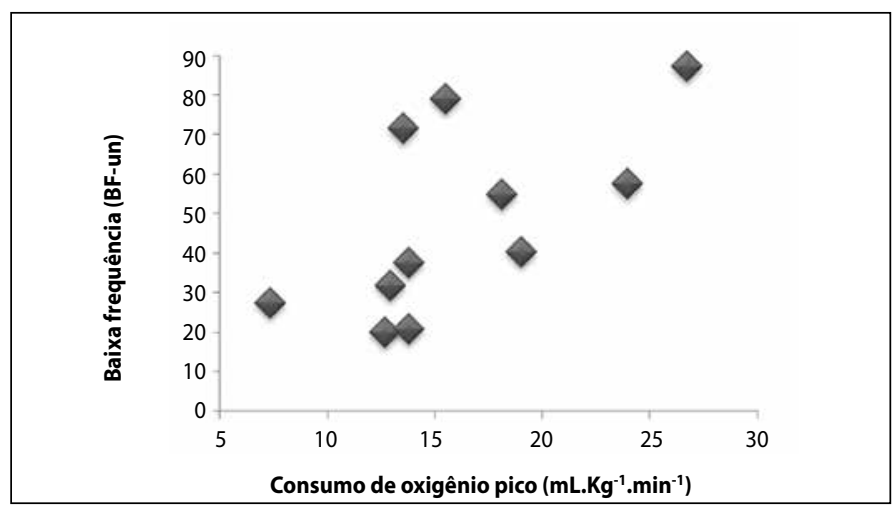

Figura 5. Dispersão do índice espectral de baixa frequência (AF - 0,15 a 0,4Hz) e do consumo pico de oxigênio $(n=11) r=0,63$ e $p=0,03$.

(eletrocardiograma, ecocardiograma e teste de esforço) e todos os testes de esforço foram interrompidos por fadiga musculoesquelética.

Essa limitação motora é decorrente dos distúrbios de contração e de tônus muscular ${ }^{2}$ e a fadiga precoce do músculo parético está associada a sua menor capacidade oxidativa ${ }^{19}$ e consequente aumento do gasto energético, decorrente da maior ativação das fibras musculares glicolíticas tipo Il e diminuição do recrutamento das fibras tipo I $^{3}$. Acredita-se que a menor capacidade da musculatura parética faz com que esses indivíduos compensem o movimento com a musculatura do membro contralateral, assim o teste de esforço aeróbico é limitado por fadiga do membro não parético, uma vez que o músculo trabalha em alta intensidade e maior concentração de lactato.

Apesar de não termos encontrado associação entre o nível de capacidade aeróbica e o uso de medicamentos, é sabido que eles podem influenciar nas respostas hemodinâmicas ao esforço físico. Os betabloqueadores limitam a elevação das variáveis hemodinâmicas por reduzir a frequência cardíaca, débito cardíaco e a pressão arteria||18,20; os bloqueadores de canais de cálcio e os inibidores da enzima conversora de angiotensina, apesar de aumentar a tolerância ao esforço ${ }^{21}$, deprimem as respostas da frequência cardíaca e da pressão arterial sistólica ${ }^{22}$.

Em relação à modulação autonômica da frequência cardíaca, no presente estudo os valores de VFC no domínio da frequência, em unidades normalizadas, se apresentaram dentro da faixa de normalidade referida pela literatura, que considera valores entre 50 a 58 para o índice de baixa frequência, que representa a atividade de ambos os eferentes com predomínio simpático, e entre 26 a 39 para alta frequência, que representa a atividade parassimpática ${ }^{23,24}$. Sabemos que não existe um consenso sobre a magnitude e o grau de acometimento neurocardíaco após o AVE, porém há estudos que contribuem em fornecer alguns parâmetros. Assim, Tokgozoglu et al.? notificaram declínio na modulação parassimpática e aumento na simpática, e Tükek et al. ${ }^{8}$ observaram diminuição em ambos eferentes. Essas adaptações são atribuídas à área e ao hemisfério cerebral acometido, responsáveis ou não pelo controle autonômico do coraçãa ${ }^{25}$. Alguns estudos afirmam que as lesões nos hemisférios cerebrais direito e esquerdo prejudicam a atividade simpática e parassimpática ${ }^{26}$, respectivamente. Contudo, não foi possível observar essa relação nessa amostra, pois participaram do estudo cinco indivíduos com lesão no hemisfério direito e seis em hemisfério esquerdo e todos apresentaram modulação autonômica da frequência cardíaca dentro da faixa de normalidade referida pela literatura.

Adicionalmente, os nossos resultados corroboram com as pesquisas que referem que na fase crônica do AVE não há alterações na modulação autonômica da frequência cardíaca ${ }^{27}$. Essa condição pode estar relacionada ao fato dessas adaptações caracterizarem uma situação transitória ${ }^{28}$ e/ou estarem relacionadas à independência funcional, ou seja, é possível que indivíduos mais independentes recuperem a VFC mais rapidamente ${ }^{29}$.

E, mesmo na presença de fatores de risco tradicionais para o AVE, como dislipidemias, hipertensão arterial e hiperglicemia, que prejudicam a modulação autonômica da frequência cardíaca ${ }^{30}$, como mostra o estudo de Hägglund et al. ${ }^{31}$ ao observar menor atividade parassimpática em indivíduos com diabetes tipo 1, neste estudo não foram observadas alterações pertinentes. Acredita-se que o uso de medicamentos para controle destes fatores de risco, como por exemplo, os betabloqueadores, os antagonistas do receptor da angiotensina 1, os alfa-adrenérgicos e os inibidores da enzima conversora de angiotensina ${ }^{20}$ associados as demandas físicas nas atividades de vida diária tenham contribuído para que a amostra apresentasse valores normais dos índices que refletem a modulação autonômica da frequência cardíaca.

Em nosso estudo foi observado que os indivíduos com maiores valores de $\mathrm{VO}_{2}$ são os menos comprometidos fisicamente, porém apresentam menor ativação parassimpática e maior simpática. Esses resultados parecem contraditórios, contudo, estes achados se assemelham ao estudo de Vieira et al..$^{32}$ que avaliaram a correlação entre a capacidade aeróbica e o controle autonômico em idosos sedentários e praticantes de atividade física, e não encontraram relação. Os autores atribuem estes resultados aos fatores que influenciam, de forma diferente, as variáveis em estudo.

No estudo de Dutra et al. ${ }^{33}$, os resultados mostram que, em sujeitos saudáveis, maiores valores de $\mathrm{VO}_{2}$ não promovem melhora adicional da modulação autonômica da frequência cardíaca, caracterizada por maior participação do componente vagal, diferente de indivíduos com doenças metabólica e cardiovascular, que respondem com melhores índices autonômicos cardíacos diante de maior capacidade aeróbica. Ou seja, quando o sistema autonômico está preservado, maiores valores de $\mathrm{VO}_{2}$ não resultará em melhor controle autonômico cardíaco. Neste sentido, os sujeitos do presente trabalho não podem ser considerados saudáveis, porém apresentam valores de VFC dentro da faixa de 
normalidade referida pela literatura e, como dissemos anteriormente, algumas pesquisas referem que na fase crônica do AVE não há alterações na modulação autonômica da frequência cardíaca ${ }^{27}$.

Por fim, este trabalho traz importantes contribuições clínicas para a avaliação e prescrição de exercício para esse tipo de paciente, uma vez observado que o consumo de oxigênio e as variáveis derivadas do teste de esforço não refletem a real capacidade do aparelho cardiopulmonar. Adicionalmente, um método simples, não invasivo e de alto valor prognóstico pode ser um recurso efetivo para avaliar o controle neurocardíaco e a saúde cardiovascular, os quais não são adequadamente exigidos em um teste de esforço físico, devido às limitações motoras.

Nossas perspectivas futuras são aumentar o número de sujeitos da amostra; a aplicação do teste cardiopulmonar para medida mais precisa do $\mathrm{VO}_{2}$ e melhor interpretação da participação dos sistemas pulmonar, cardiovascular e musculoesquelético. Por fim, incluir análise da independência funcional para investigar se as adaptações autonômicas cardíacas estão relacionadas à mesma. Já as limitações do estudo referem-se ao número de sujeitos da amostra que ficou abaixo do indicado pelo cálculo amostral em virtude das dificuldades em encontrar pessoas que preenchessem os critérios de seleção; a impossibilidade de acesso ao exame diagnóstico do AVE para melhor determinar a área de lesão; e a falta de um grupo controle com o perfil similar ao estudado.

\section{CONCLUSÃO}

De acordo com nosso estudo é possível concluir que na fase crônica do AVE, a modulação autonômica da frequência cardíaca encontra-se normal e aqueles indivíduos com menor capacidade aeróbica apresentam maior modulação parassimpática e menor simpática, provavelmente em consequência da maior exigência física nas atividades de vida diária decorrente da maior limitação motora.

\section{AGRADECIMENTOS}

Os autores agradecem ao Instituto do Coração de Marília (ICM), o Laboratório de Análise Clínica São Francisco, aos órgãos de fomento, Conselho Nacional de Desenvolvimento Científico e Tecnológico (CNPQ) e Fundação para o Desenvolvimento da Unesp (FUNDUNESP)., Centro de Educação e Estudos em Saúde (CEES) e à Secretária de Saúde Pública do Município de Marília/SP.

Todos os autores declararam não haver qualquer potencial conflito de interesses referente a este artigo.

CONTRIBUIÇÕES DOS AUTORES: Cada autor contribuiu individual e significativamente para o desenvolvimento do manuscrito. MFC (0000-0001-5110-8431)* e SV (0000-0003-4241-1687)* foram as principais contribuintes na redação do manuscrito, responsáveis pela revisão bibliográfica, testes pilotos, triagem e avaliação dos indivíduos, análise dos dados. RJQ (0000-0003-4241-1687)* foi o idealizador do projeto e dos protocolos, orientação da pesquisa, análise dos dados, orientação e correção do manuscrito. AMC (0000-0002-4837-3936)* e ARPA (0000-0002-1807-1953)* colaboraram na análise e correção do manuscrito. *ORCID (Open Researcher and Contributor ID).

\section{REFERÊNCIAS}

1. Zorowitz RD, Gross E, Polinski DM. The stroke survivor. Disabil Rehabil. 2002;24(13):666-79.

2. Fernandes MA, Freitas BHPF, Negrini F, Sampaio LMM, Medalha CC. Contribuições da avaliação cardiorrespiratória em pacientes hemiplégicos. Arq Sanny Pesq Saúde. 2008;1(2):90-7.

3. Jorgensen JR, Bech-Pedersen DT, Zeeman P, Sørensen J, Andersen LL, Schonberger M. Effect of intensive outpatient physical training on gait performance and cardiovascular health in people with hemiparesis after stroke. Phis Ther. 2010;90(4):527-37.

4. Teixeira-Salmela LF, Oliveira ESG, Santana EGS, Resende GP. Fortalecimento muscular e condicionamento físico em hemiplégicos. Acta Fisiátr. 2000;7(3):108-18.

5. Oppenheimer SM, Gelb A, Girvin JP, Hachinski VC. Cardiovascular effects of human insular cortex stimulation.Neurology.1992;42(9):1727-32.

6. Ovando AC, Michaelsen SM, Carvalho Td, Herber V. Avaliação da aptidão cardiopulmonar em indivíduos com hemiparesia após acidente vascular encefálico. Arq Bras Cardiol. 2011;96(2):140-7.

7. Tokgozoglu SL, Batur MK, Topçouglu MA, Saribas O, Kes S, Oto A. Effects of stroke localization um cardiac autonomic balance and sudden death. Stroke. 1999;30(7):1307-11

8. Tükek T, Yildiz P, Atilgan D, Tuzcu V, Eren M, Erk $\mathrm{O}$, et al. Effect of diurnal variability of heart rate on development of arrhythmia in patients with chronic obstructive pulmonary disease. Int J Cardiol. 2003:88(2-3):199-206.

9. Lakusic N, Mahovic D, Babic T. Gradual recovery of impaired cardiac autonomic balance within first six months after ischemic cerebral stroke. Acta Neurol Belg. 2005;105(1):39-42.

10. Fronchetti L, Aguiar CA, Aguiar AF, Nakamura FY, De-Oliveira FR. Modificações da variabilidade da frequência cardíaca frente ao exercício e treinamento físico. R Min Educ Fis. 2007:15(2):101-29.

11. Buchheit M, Chivot A, Parouty J, Mercier D, Al Haddad H, Laursen PB, et al. Monitoring endurance running performance using cardiac parasympathetic function. Eur J Appl Physiol. 2010;108(6):1153-67.

12. Meirelles LR, Pinto VLM, Medeiros AS, Berry JRS, Magalhães CK. Efeito da atividade física supervisionada após 6 meses de reabilitação cardíaca: experiência inicial. Revista da SOCERJ. 2006;19(6):474-81.

13. Kawamura T. Avaliação da capacidade física e teste ergométrico. Rev Soc Cardiol SP. 2001;11(3):659-72.

14. Leite PF. Fisiologia do exercício, ergometria e condicionamento físico. In: Leite PF. Cardiologia desportiva. 4 ed. São Paulo: Robe Editorial, 2000. p. 37-38

15. Gamelin FX, Berthoin S, Bosquet L. Validity of Polar $\$ 810$ Heart Rate Monitor to Measure R-R Intervals at Rest. Med Sci Sports Exerc.2006;38(5):887-93.

16. Longo A, Ferreira D, Correia MJ. Variabilidade da frequência cardíaca. Rev Port Cardiol.1995;14(3):241-62.

17. Pang MYC, Eng JJ, Dawson AS. Relationship between ambulatory capacity and cardiorespiratory fitness in chronic stroke: influence of stroke-specific impairments. Chest. 2005;127(2):495-501
18. Rocha JP, Schuster RC, Polese JC, Mazzola D, Oliveira SG. Análise da capacidade funcional de idosos hemiparéticos crônicos: estudo de caso. Man. 2010;8(S1):68-74

19. Jakovljevic DG, Moore SA, Tan LB, Rochester L, Ford GA, Trenell MI. Discrepancy between cardiac and physical functional reserves in stroke. Stroke. 2012;43(5):1422-25.

20. Opie LH, Gersh BJ. Fármacos em Cardiologia. 6a ed. Rio de Janeiro: Di Livros, 2007.

21. Carreira MA, Tavares LR, Leite RF, Ribeiro JC, Santos AC, Pereira KG et al. Exercise Testing in Hypertensive Patients Taking Different Angiotensin-Converting Enzyme Inhibitors. Arq Bras Cardiol. 2003;80(2):133-7.

22. Kostis JB, Shindles DM, Moreyra AE, Ruddy MC, Schneider S, Cosgrove N. Differential exercise effects of captopril and nadolol in patients with essential hypertension. Angiology. 1992;43(8):647-52.

23. Heart rate variability. standards of measurement, physiological interpretation, and clinical use. circulation. Task Force of the European Society of Cardiology of the North American Society of Pacing Electrophysiology. 1996;93(5):1043-65.

24. Nunan D, Sandercock GRH, Brodie DA. A quantitative systematic review of normal values for short-term heart rate variability in healthy adults. Pacing Clin Electrophysiol;2010;33(11):1407-17.

25. He L, Li C, Luo Y, Dong W, Yang H. Clinical prognostic significance of heart abnormality and heart rate variability in patients with stroke. Neurol Res. 2010;32(5):530-4

26. Lane RD, Wallace JD, Petrosky PP, Schwartz GE, Gradman AH. Supraventricular tachycardia in patients with right hemisphere strokes. Stroke.1992;23(3):362-6.

27. Lakusic N, Mahovic D, Babic T. Gradual recovery of impaired cardiac autonomic balance within first six months after ischemic cerebral stroke. Acta Neurol Belg. 2005;105(1):39-42.

28. Meglic B, Kobal J, Osredkar J, PogacnikT. Autonomic nervous system function in patients with acute brainstem stroke. Cerebrovasc Dis. 2001;11(1):2-8.

29. Bassi A, Colivicchi F, Santin M, Caltagirone C. Cardiac autonomic dysfunction and functional outcome after ischaemic stroke. Eur J Neurol.. 2007;14(8):917-22.

30. Shehab A, Elnour AA, Struthers AD. Heart rate variability as an indicator of left ventricular systolic dysfunction. Cardiovasc J Afr. 2009;20(5):278-83.

31. Hägglund H, Uusitalo A, Peltonen JE, Koponen AS, Aho J, Tiinanen S, et al. Cardiovascular autonomic nervous system function and aerobic capacity in type 1 diabetes. Front Physiol. 2012;3(356):1-8.

32. Vieira S, Felix ACS, Quitério RJ.Variabilidade da frequência cardíaca e carga máxima atingida no teste de esforço físico dinâmico em homens idosos. Rev Bras Med. Esporte 2012;18(6):377-80.

33. Dutra SG, Pereira AP, Tezini GC, Mazon JH, Martins-Pinge MC, Souza HC. Cardiac autonomic modulation is determined by gender and is independent of aerobic physical capacity in healthy subjects. Plos One. 2013:8(10):1-9. 